\title{
Mechanically tuneable microoptical structure based on PDMS
}

\author{
V.J. Cadarso ${ }^{\mathrm{a}, \mathrm{b}, *}$, A. Llobera ${ }^{\mathrm{a}}$, G. Villanueva ${ }^{\mathrm{a}}$, J.A. Plaza ${ }^{\mathrm{a}}$, J. Brugger $^{\mathrm{b}}$, C. Dominguez $^{\mathrm{a}}$ \\ a Instituto de Microelectrònica de Barcelona (IMB-CNM-CSIC), Campus UAB 08193 Barcelona, Spain \\ ${ }^{\mathrm{b}}$ Ecole Polytechnique Federale de Lausanne (EPFL), CH-1015 Lausanne, Switzerland
}

\section{A R T I C L E I N F O}

\section{Article history:}

Received 30 September 2009

Received in revised form 25 February 2010

Accepted 26 February 2010

Available online 6 March 2010

\section{Keywords:}

Microlenses

Tuneable focus

PDMS

\begin{abstract}
A B S T R A C T
A system of two solid microlenses with uncoupled optical properties is presented. This structure has been designed in order to have one lens as a reference, while the other one can be mechanically tuneable. The reference lens presents a diameter of $2 \mu \mathrm{m}$ and it is placed in the optical axis of the mechanically tuneable lens, which has a diameter of $10 \mu \mathrm{m}$. The proposed microoptical structure has been fabricated in poly(dimethilsiloxane) (PDMS) merging deep reactive ion etching, SU-8 and soft lithography, with a low-cost (mass-production), simple and highly repetitive technology. This device was numerically simulated prior to its fabrication, to optimize its design and improve its behaviour. In addition, an optical characterization of the fabricated devices was carried out. Both simulation and experimental results shows a good agreement, under mechanical actuation behaviour of the reference lens is invariable, while the tuneable lens become an elliptic lens and the interval of Sturm can be observed. These results provide a proof of concept of the proposed devices and validate both the design and the fabrication technology. (c) 2010 Elsevier B.V. All rights reserved.
\end{abstract}

\section{Introduction}

Development of microlenses has become a major research field during the last years. Such components are used in a myriad of applications, such as in lab-on-a-chip systems [1,2], enhancement of LEDs out-coupling efficiency [3,4], fluorescence sensors [5], fibre coupling [6], scanning [7] or imaging [8]. Several approaches have been proposed in order to obtain the microlenses. Ink jet printing [9], hot embossing [10,11], photoembossing [12], soft lithography [13] and photothermal patterning [14] are some examples of techniques used to develop such components, being moulding the most used method, since it allows the development of the optical structures in different materials assuring high repeatability and low cost. Nevertheless, these techniques must be adapted and optimized to fulfil all the requirements of the different applications. Concretely, microlenses with variable focal length have devoted a great interest since using such systems the need for optical alignment or scanning is eliminated. Although these structures have been successfully developed, usually present several drawbacks. Most common approaches are based on a thin membrane that requires complex heating [15] or microfluidic systems [16,17] to achieve the actuation over the lenses; resulting on fragile devices.

Due to the interesting properties of poly(dimethilsiloxane) (PDMS), it has been used for developing modulable optical systems,

\footnotetext{
* Corresponding author at: Ecole Polytechnique Federale de Lausanne (EPFL), CH1015 Lausanne, Switzerland. Tel.: +41 021693 7818; fax: +41 0216936670.

E-mail address: victor.cadarso@epfl.ch (V.J. Cadarso).
}

either using diffraction gratings [18], fillable lenses[17], or optical cantilevers with integrated microlenses [19]. Its high transmittance from the visible to the near infrared, its very low Young's modulus (between 300 and $1760 \mathrm{kPa}[20,21]$ ), which permits obtaining large structures with low spring constant, its low cost, and its technological simplicity can be highlighted. In this work, in an attempt to solve the previously mentioned drawbacks of the membranebased microlenses, the mechanical and optical properties of PDMS have been taken in advantage to define a mechanically tuneable microoptical structure. The proposed system is not based on membranes with the aim of obtain a robust structure, and the actuation is mechanically induced, avoiding the use of complex actuation systems. The microoptical structure consists on two microlenses with uncoupled optical properties. This system allow to improve the capabilities of the existing tuneable microlenes, since it has been designed and numerically simulated, to assure that one of the lenses can be used as a reference lens (with invariable focal properties) while the other one is easily tuneable. The fabrication technology is based in a double mastering process that merges silicon micromechanization, SU-8 lithography and soft lithography, allowing a simple and low-cost fabrication of this complex system. Such properties make the proposed microoptical structure as an interesting candidate for the development of lab-on-a-chip and low-cost tuneable imaging scanning systems.

\section{Design and simulation}

The proposed configuration is presented on Fig. 1. The small lens $(\mathrm{Sl})$ or reference lens is placed at the centre of the large lens 


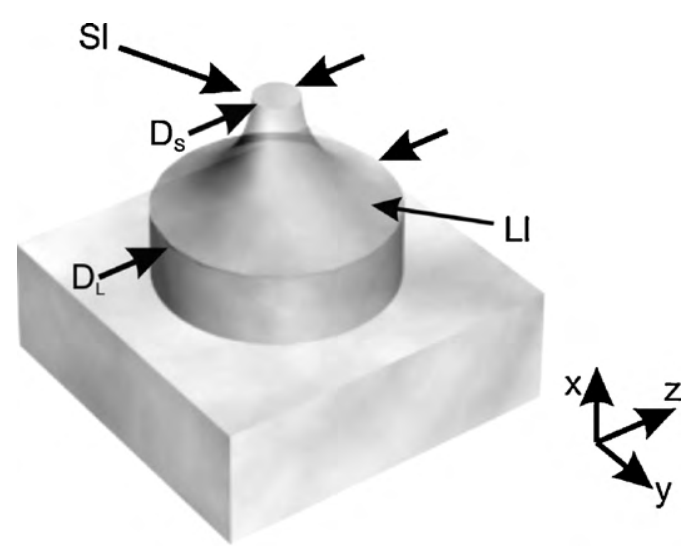

Fig. 1. Schema of the proposed microoptical system showing all its components, the small lens ( $\mathrm{Sl}$ ) with a diameter of DS and the large lens ( $\mathrm{Ll}$ ) with a diameter of DL.

(Ll) or scanning lens conforming a solid system and therefore both focuses are on the same optical axis. Moreover, since the light passes through both lenses simultaneously and not consecutively, the microlens system can be considered as uncoupled. With the aim to obtain these optical properties, the diameter of the Sl (DS) has been fixed at the minimum value possible with the standard UV photolithographic techniques; namely DS has been fixed at $2 \mu \mathrm{m}$. On the other hand, the $\mathrm{Ll}$ has been designed to assure that the different focuses can be clearly distinguished. Hence, the diameter of the $\mathrm{Ll}$ (DL) has been fixed at $10 \mu \mathrm{m}$.

Due to the complexity of this design a numerical analysis has been done using FIMMWAVE and FIMMPROP software (Photon Design, UK), a Gaussian beam illumination $(\lambda=670 \mathrm{~nm})$ has been considered. Optical behaviour of the system is schematized in Fig. 2a when no actuation force is considered. Simulations predict that the light is focused on two different planes: a first focus in the Sl focal plane for the light focused from the Sl, and a second focus in the $\mathrm{Ll}$ plane of the light focused from the Ll. Moreover, simulations show that first and second focus patterns have revolution symmetry and both are surrounded by a ring. For the first focus the ring is labelled as primary ring (PR) and it is due to the light converging from the Ll. So, when the light of the PR continues propagating it will converge and become the second focus point. Conversely, on the $\mathrm{Ll}$ focal plane the second focus is surrounded by the secondary ring (SR), which is the light that diverges from the first focus. Therefore, the PR and the second focus point should have the same power and, on the other hand, the power of the first focus point and the power of the SR should match as well. Simulations have shown that for the PR and the second focus the power is 0.3 a.u., while for the SR and the first focus the power is 0.1 a.u. This result clearly shows that the behaviour of both lenses is uncoupled since the light that is focused by one of them does not interact with the light focused by the other one.

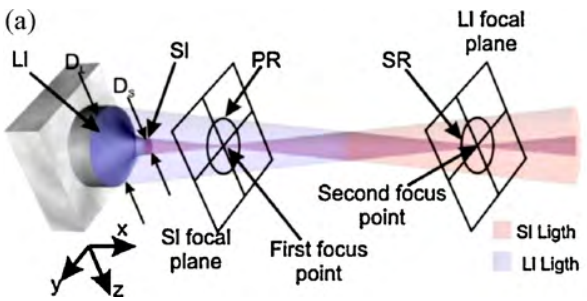

As it was advanced, such a system can be easily actuated mechanically. When a uniaxial actuation force is applied over the whole structure, the Sl does not suffer measurable variation of its curvature. In opposition, the $\mathrm{Ll}$ will be stretched due to its larger diameter. So, thanks to the mechanical properties of the PDMS, it is easy to modify the shape of the $\mathrm{Ll}$ microlens stretching the structure by horizontal mechanical actuation (on the $y$-axis). Using this method it is possible to modulate their behaviour; conceptual scheme of this can be seen on the Fig. 2b. This behaviour under a stretching of $10 \%$ in the $y$-axis has been analyzed using also FIMMWAVE and FIMMPROP. Since the diameter of the Sl is increased only on $200 \mathrm{~nm}$, the behaviour of the first focus is not modified and simulations predict that revolution symmetry is conserved. Nevertheless, the PR is significantly modified, but this is consistent since the PR is a result of the light focusing from the $\mathrm{Ll}$ and its diameter is increased in $1 \mu \mathrm{m}$ on the $y$-axis. This results in a drastic variation of the $\mathrm{Ll}$ behaviour and loses its revolution symmetry, since it becomes an elliptic lens. Hence the first focus point will remain unaltered whereas, under stretching, the second focus is converted to an interval of Sturm [22]. Three elements can be observed on this interval. On a surface at one end of the interval is the horizontal line focus (HLF), on the other surface end is the vertical line focus (VLF), and where the image is least blurred is the circle of least confusion (CLC). This behaviour is usual on an astigmatic lens and it is due to the different curvatures on the $y$ - and $z$-axes. These results validate the use of the $\mathrm{Sl}$ as a reference lens, while the $\mathrm{Ll}$ can be used as a scanning or sensing lens.

\section{Fabrication}

Combination of silicon micromachining technologies with SU-8 technologies and soft lithography techniques to achieve microsystems based on PDMS has been previously demonstrated. Nevertheless, the pattern to be transferred to the PDMS using this hybrid technology is usually very simple, such as microcapillaries to perform a vent valve for handle picoliter liquid samples [23], diffraction gratings for pressure monitoring [24] or multiple internal reflection systems [25]. However, the use of such a mixed technology to develop the complex microlens system proposed with a simple method is possible. This mixed technology can be divided on three stages: silicon micromechanization with deep reactive ion etching (DRIE) to develop the complex 3D structure of the micooptical system; SU-8 technology to perform the soft lithography master with the negative of the system; and the cast moulding soft lithography technique [26] to transfer the pattern to the PDMS and obtain the microoptical systems.

Processing of the PDMS-based optical microsystems are carried over a $(100)$ oriented silicon wafer of $4^{\prime \prime}$ diameter and $525-\mu \mathrm{m}$ thickness. In order to perform the mask on the silicon micromechanization process the wafer is wet oxidized up to 400 -nm thick and a photolithographic step is carried out with the only one mask needed to perform the microoptical systems, shows in Fig. 3a. Then a nearly isotropic etch is done by DRIE to obtain a sharp apex. To

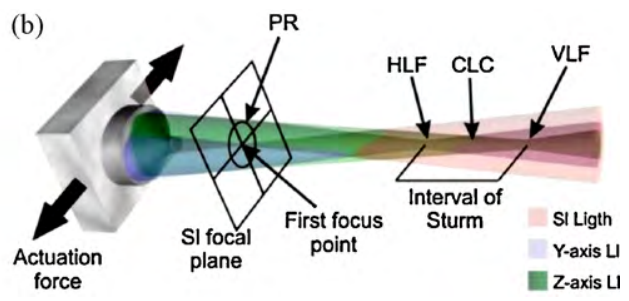

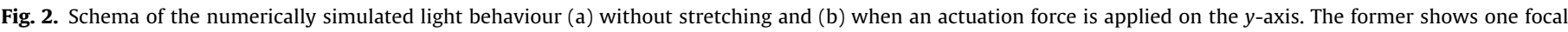

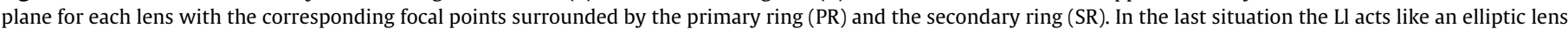
showing the interval of Sturm with all its elements: the horizontal line focus (HLF), the circle of least confusion (CLC) and the vertical line focus (VLF). 

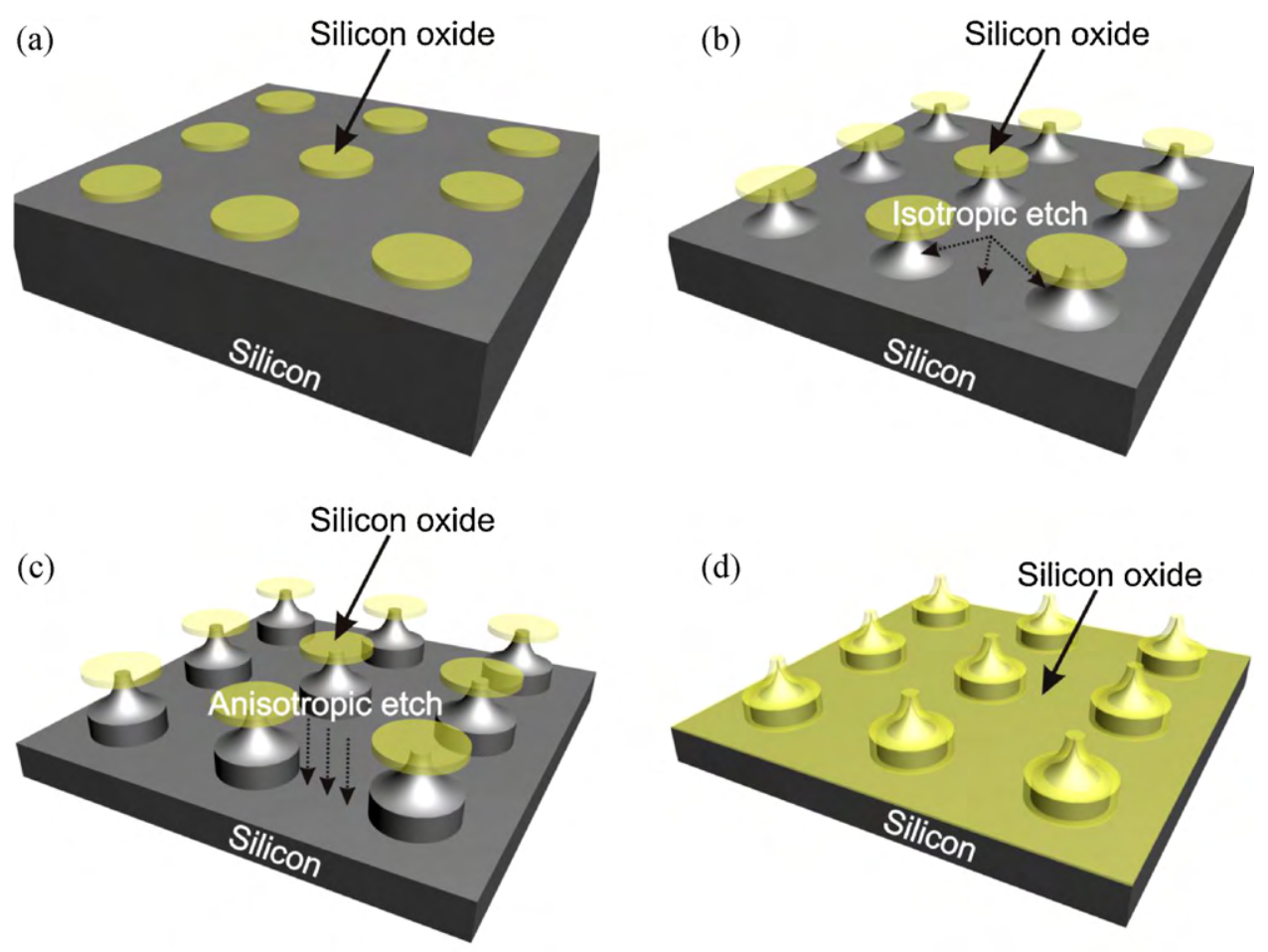

(e)
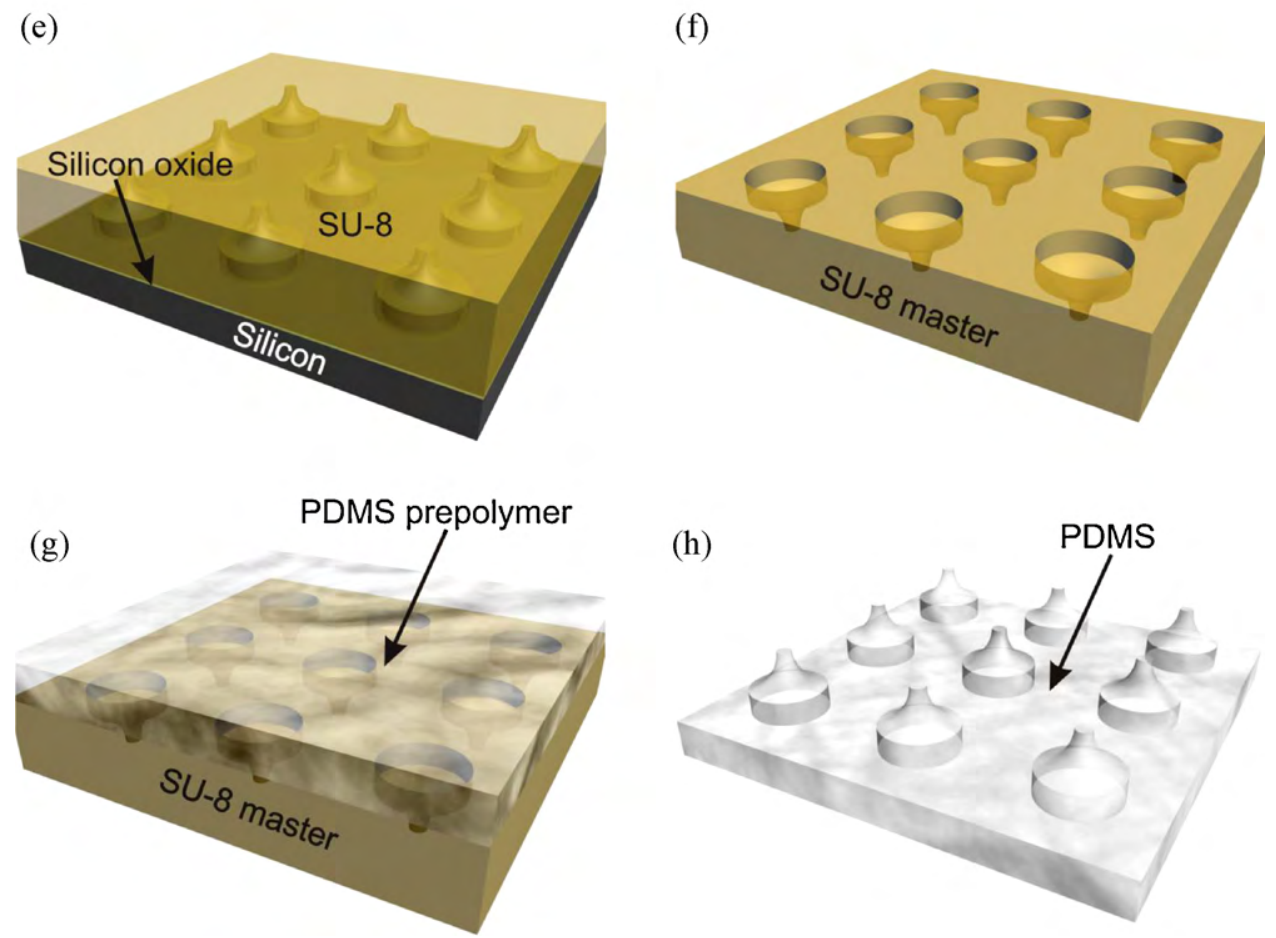

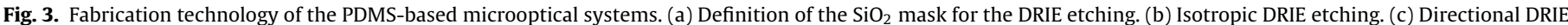

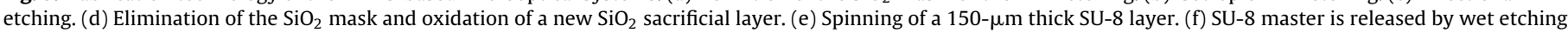
of the $\mathrm{SiO}_{2}$ sacrificial layer. (g) Cast moulding of the PDMS pre-polymer over the SU-8 master. (h) After curing the PDMS is mechanically released.

this effect an etching cycle is done for $120 \mathrm{~s}$, without any passivation cycle (see Fig. 3b). After that, in the same DRIE step, etching conditions are modified so as to obtain a vertical etch profile process, as it is schematized in Fig. 3c. Hence, a stage alternating between etching and passivation cycles is done for $90 \mathrm{~s}$. Then, the $\mathrm{SiO}_{2}$ mask is removed by wet etching and a new $\mathrm{SiO}_{2}$ layer of $400-\mathrm{nm}$ thick is thermally growth to be used as a sacrificial layer. After this step, the shape of the proposed microoptical system is fully defined in the $\mathrm{SiO}_{2} / \mathrm{Si}$ structure, as can be observed at Fig. 3d, and the Si microme- canization stage is finished. At this point this $\mathrm{SiO}_{2} / \mathrm{Si}$ master is used to define a SU-8 master over which the PDMS cast moulding will be done to obtain the proposed system. This "double mastering" process starts with the spinning of a layer of $150-\mu \mathrm{m}$ thick SU-8. Then a soft-bake at $65^{\circ} \mathrm{C}$ for $10 \mathrm{~min}$ is done, followed by $95^{\circ} \mathrm{C}$ for $2 \mathrm{~h}$. After the wafer returns to room temperature it is exposed $60 \mathrm{~s}$ to UV without any kind of mask and a post exposure bake (PEB) of $30 \mathrm{~min}$ at $95^{\circ} \mathrm{C}$ is done, as it is shown in Fig. 3e. After the PEB, the wafer is immersed in $\mathrm{HF}$ in order to etch the $\mathrm{SiO}_{2}$ sacrificial layer 

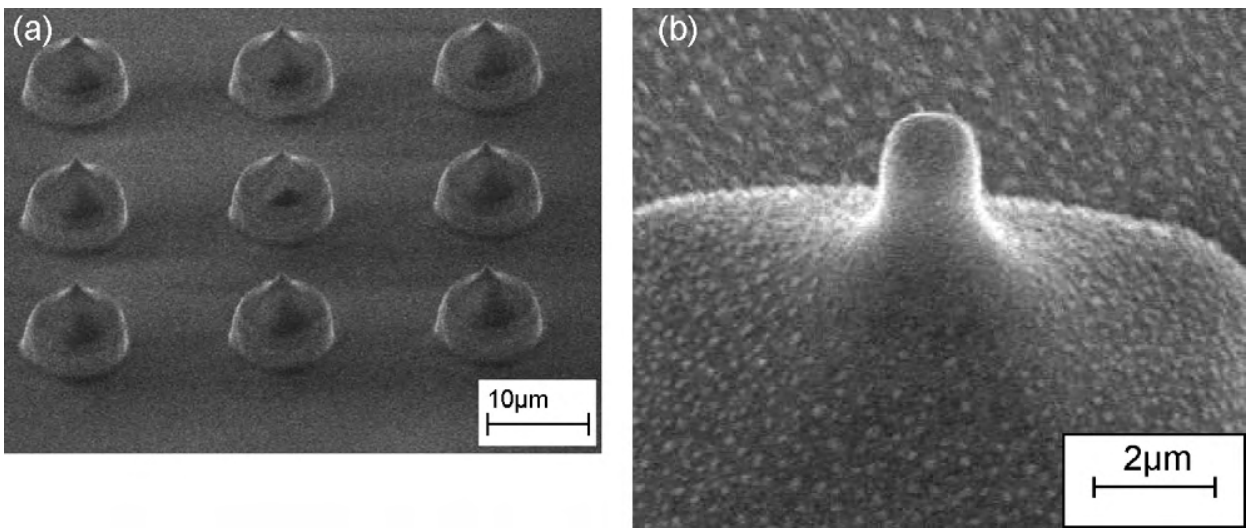

Fig. 4. (a) SEM images of a $3 \times 3$ matrix PDMS microoptical systems and (b) detail of one small lens placed over the large lens.

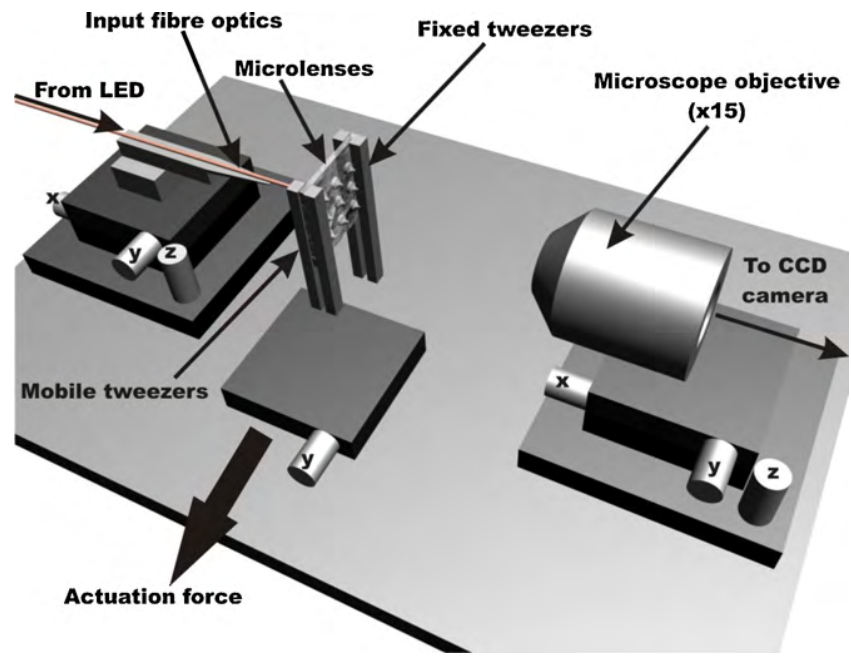

Fig. 5. Schema of the experimental set up used in the characterization of the proposed microlenses.

and release the patterned SU-8 layer from the silicon substrate, as it is shown in Fig. 3f. As it has been previously mentioned, this SU-8 layer will be used as a master on the final cast moulding process to transfer the pattern of the proposed microoptical system to the PDMS. The PDMS (Sylgard 184 elastomer kit, Dow Corning, Midland, MI, USA) pre-polymer is obtained by mixing the curing agent with the elastomer base in a $1: 10$ ratio $(\mathrm{v}: \mathrm{v})$. The subsequent mixture is degassed to remove the air bubbles, casted over the master, as it is schematized in Fig. $3 \mathrm{~g}$, and degassed again to assure that microcavities are filled without any residual air. Once the PDMS is polymerized, the microoptical system is mechanically released from the SU- 8 master and the fabrication of the optical microsystem is finished, Fig. 3h. At this point, it is possible to repeat final cast moulding steps several times with the same SU-8 master to obtain a large number of systems.

Fig. 4a shows a SEM picture of a $3 \times 3$ array of the fabricated microoptical systems, while Fig. $4 \mathrm{~b}$ shows the detail of one small lens over the large lens. As it can be seen on these pictures, the
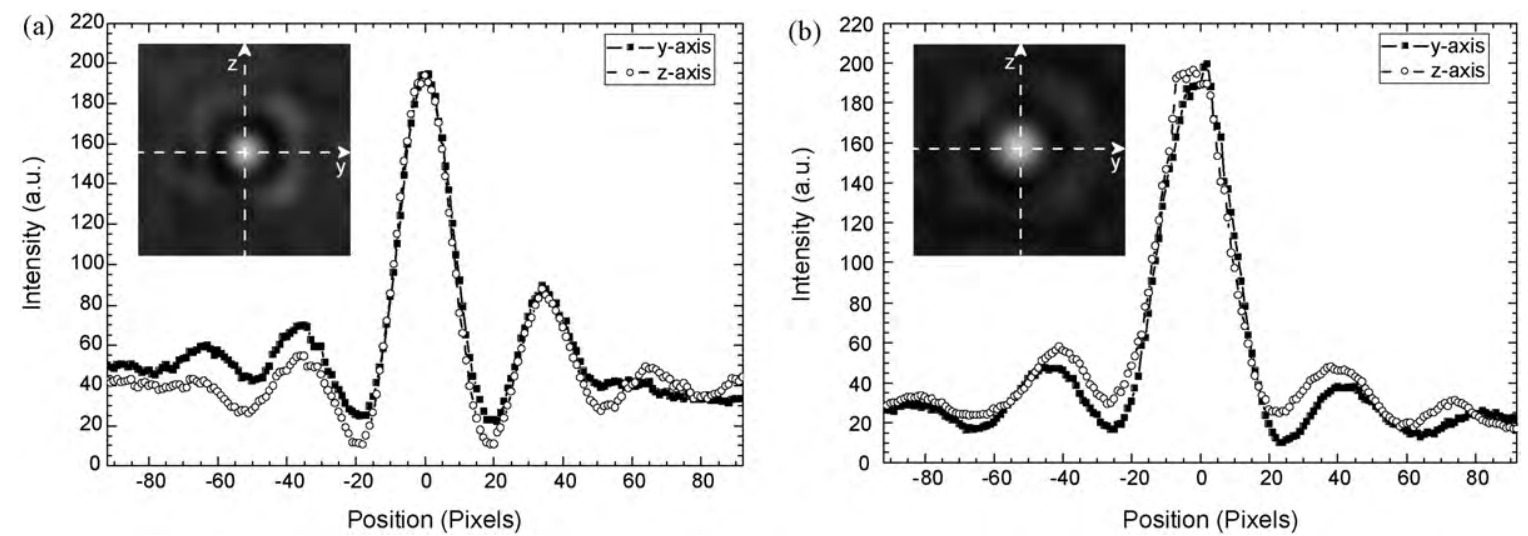

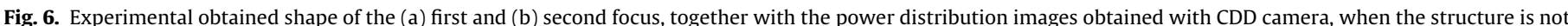
stretched. 

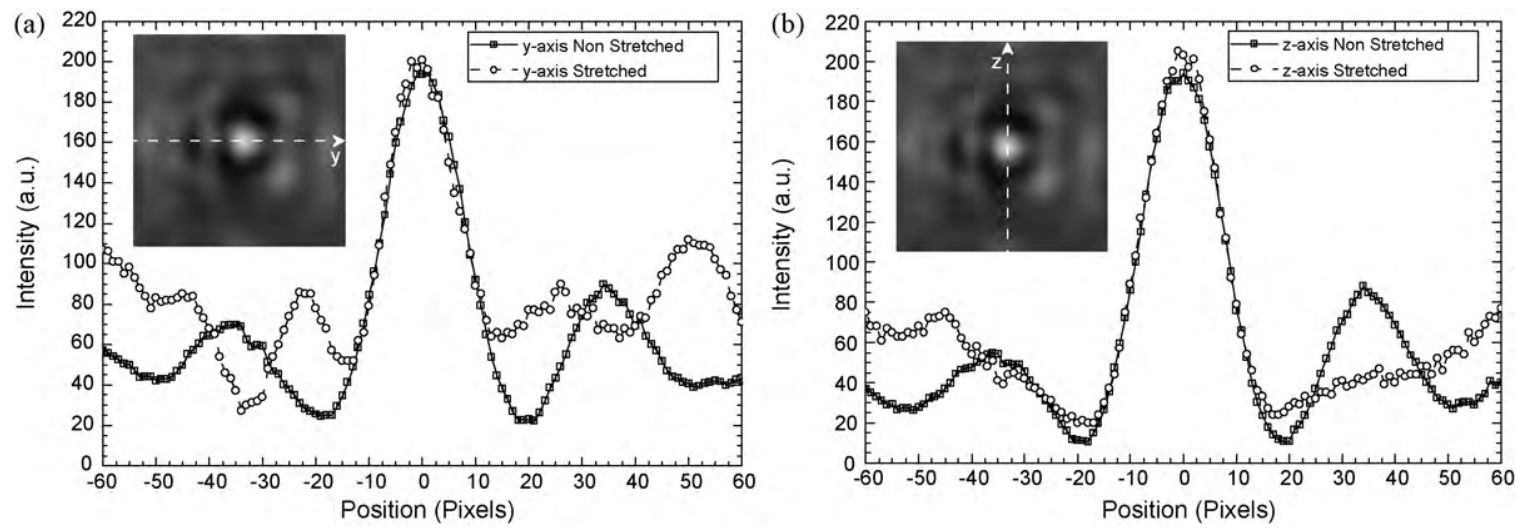

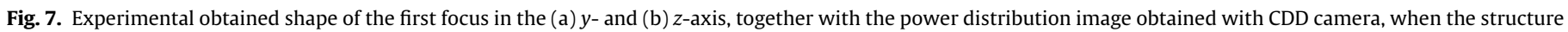
is stretched. The profiles of the first focus when no actuation is applied are also represented.
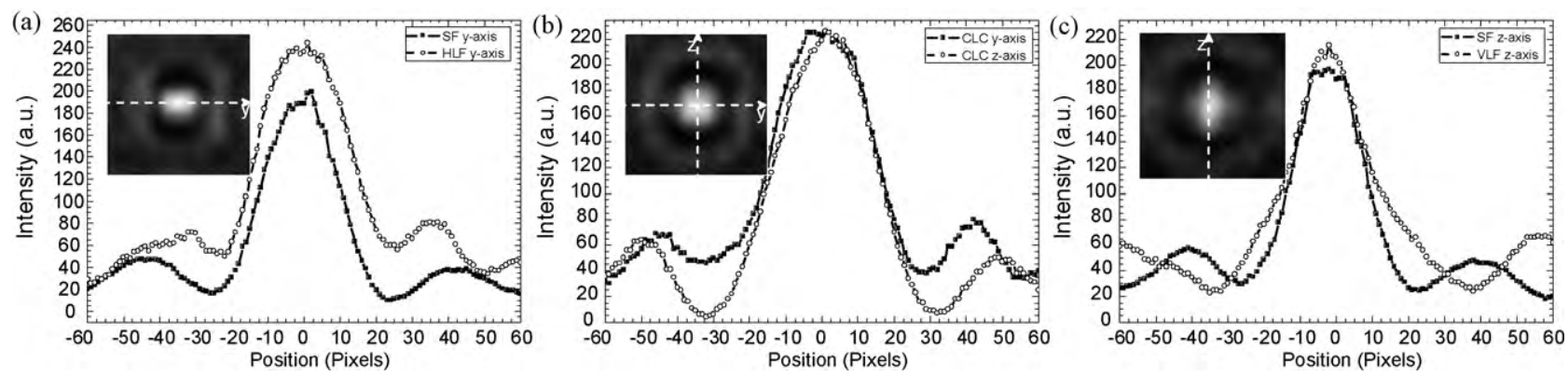

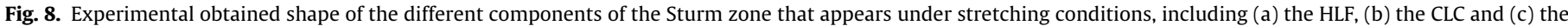
VLF. Insets show the CDD images obtained of the different elements.

fabricated microoptical systems show a high quality and repeatability. The slight roughness that can be observed is a result of the gold coating layer used to obtain the SEM images. These results validate the proposed mixed technology which, although it is based on a non-standard double moulding process, it only requires one photolithographic mask and after the definition of the SU-8 master, it is simplified to a typical cast moulding process.

\section{Characterization}

Optical characterization of the proposed PDMS-based microoptical system has been made using the experimental set up schematized in Fig. 5: a multimode fibre with a core diameter of $50 \mu \mathrm{m}$ connected to a LED with a working wavelength of $670 \mathrm{~nm}$. In order to assure the correct positioning of this fibre it is placed over an $x-y-z$ micropositioner with a resolution of $2 \mu \mathrm{m}$. The microoptical system is held with two tweezers. The first one is fixed throughout the experiment. Conversely, the second one is placed over a $y$ micropositioner in order to stretch the structure, and perform the modulation. A microscope objective $(\times 15)$ placed over a $x-y-z$ micropositioner with a resolution of $2 \mu \mathrm{m}$ is used to collect the output light and focus it on a CCD camera Pixelfly 200 XS VGA (pco.imagin, Kelheim, Germany) connected to a computer.

Initially, the microoptical system is studied without applying any actuation force. Shapes of the first and the second focus, in $y$ and $z$-axis, are presented in Fig. $6 \mathrm{a}$ and b, respectively. The insets show the experimental power distribution, obtained with the CCD camera. As can be seen both focuses show revolution symmetry and are surrounded by a ring of light. Moreover, $\mathrm{Ll}$ is focusing more light than $\mathrm{Sl}$ (as it can be noticed in the difference of width), since calculated power for the first focus and second focus is $3802 \pm 158$ a.u. and $5282 \pm 273$ a.u., respectively. This behaviour was predicted by the simulations.
After confirm the expected behaviour of the proposed microlens system without any actuation, the mobile tweezers is displaced so as to cause a stress on the structure and therefore convert the $\mathrm{Ll}$ into an elliptical microlens. Under a stretching of $10 \%$ behaviour of the first focus is not modified and revolution symmetry is conserved. Hence, as it is observed on the Fig. 7, the shape on the focus point for the (a) $y$ - and (b) $z$-axes is equivalent to that observed for the non-stretched lens (also presented in this figure). On the other hand, the surrounding ring has been drastically modified. These results were predicted by the simulations and confirm that under stretching conditions the Sl lens remains invariable while the $\mathrm{Ll}$ is significantly modified.

Conversely, since the $\mathrm{Ll}$ becomes an elliptic lens it is not possible to find the second focus under stretching conditions. Instead, the elements of the interval of Sturm are easily observed. Horizontal line focus, can be observed in the inset of Fig. 8a, as it can be observed, the width is increased in the $y$-axis when it is compared with the presented for the non-actuated second focus. The circle of least confusion is presented in Fig. 8b, in this case the shape in both axes is similar as it was expected. Finally, the vertical line focus can be observed in Fig. $8 c$, in this case the width is increased in the $z$-axis.

The presented results confirm both the technology and the principle of operation, and validate the use of the Sl as a reference lens, sine under stretching conditions it remains invariable, and the use of the $\mathrm{Ll}$ as the scanning or sensing lens, since this behaviour can be tuned in stretching conditions.

\section{Conclusions}

A mechanically tuneable microoptical system has been designed, simulated, fabricated and characterized. The proposed system is based in two optically uncoupled lenses, one used as a ref- 
erence lens, while the other one can be used as a scanning or sensing lens. Numerical simulations have shown that when no actuation is applied the light is focused on two different focuses, which present revolution symmetry. Conversely, when an actuation is applied the $\mathrm{Sl}$ rest invariable while the $\mathrm{Ll}$ becomes an elliptic lens and forms the interval of Sturm. Fabrication of this complex system has been achieved easily merging DRIE silicon micromachining, SU-8 lithography and a soft lithography, allowing the development of the full structure with the use of only one single photolithographic mask. Moreover, after the fabrication of the SU-8 master the process is reduced to one single step, which can be repeated several times over the same master assuring a high repeatability. Experimental results have shown a good agreement with simulations, proving that the $\mathrm{Sl}$ can be used as reference lens and the $\mathrm{Ll}$ forms the interval of Sturm under stretching conditions. This structure is an interesting candidate for applications on photonic imaging, sensing and lab-on-a-chip systems.

\section{Acknowledgements}

This work has been made with funds of the project MINAHE 2 (TEC2005-07996-C02-01) and the project 4Senses (PSE- 0204002007-1); both of them financed by the Spanish Ministerio de Educación y Ciencia (MEC). The authors thank to Photon Design for his help and fruitful discussions. V.J.C. acknowledges the Consejo Superior de Investigaciones Científicas (CSIC) the grant conceded by the program I3P, cofinancing by the European Social Fund. A.L. acknowledges the Spanish Ministry of Science and Education for the award of a Ramón y Cajal contract.

\section{References}

[1] J. Seo, L.P. Lee, Disposable integrated microfluidics with self-aligned planar microlenses, Sensors and Actuators B-Chemical 99 (2-3) (2004) 615-622.

[2] R.P.J. Barretto, B. Messerschmidt, M.J. Schnitzer, In vivo fluorescence imaging with high-resolution microlenses, Nature Methods 6 (7) (2009) 511-561.

[3] D.F. Kuang, X.L. Zhang, M. Gui, Z.L. Fang, Hexagonal microlens array fabricated by direct laser writing and inductively coupled plasma etching on organic light emitting devices to enhance the outcoupling efficiency, Applied Optics 48 (5) (2009) 974-978.

[4] M. Khizar, Z.Y. Fan, K.H. Kim, J.Y. Lin, H.X. Jiang, Nitride deep-ultraviolet lightemitting diodes with microlens array, Applied Physics Letters 86 (17) (2005)

[5] K.Y. Hung, F.G. Tseng, Integrated three-dimensional optical MEMS for chip-based fluorescence detection, Journal of Micromechanics and Microengineering 19 (4) (2009) 10

[6] C.A. Edwards, H.M. Presby, C. Dragone, Ideal microlenses for laser to fiber coupling, Journal of Lightwave Technology 11 (2) (1993) 252-257.

[7] T. Tanaami, S. Otsuki, N. Tomosada, Y. Kosugi, M. Shimizu, H. Ishida, High-speed 1 -frame/ms scanning confocal microscope with a microlens and Nipkow disks, Applied Optics 41 (22) (2002) 4704-4708.

[8] M. Martinez-Corral, B. Javidi, R. Martinez-Cuenca, G. Saavedra, Integral imaging with improved depth of field by use of amplitude-modulated microlens arrays, Applied Optics 43 (31) (2004) 5806-5813.

[9] S. Biehl, R. Danzebrink, P. Oliveira, M.A. Aegerter, Refractive microlens fabrication by ink-jet process, Journal of Sol-Gel Science and Technology 13 (1-3) (1998) 177-182.

[10] N.S. Ong, Y.H. Koh, Y.Q. Fu, Microlens array produced using hot embossing process, Microelectronic Engineering 60 (3-4) (2002) 365-379.

[11] X.J. Shen, L.W. Pan, L.W. Lin, Microplastic embossing process: experimental and theoretical characterizations, Sensors and Actuators a-Physical 97 (8) (2002) 428-433.

[12] K. Hermans, S.Z. Harnidi, Rapid, direct fabrication of antireflection-coated microlens arrays by photoembossing, Applied Optics 47 (35) (2008) 6512-6517.

[13] M.V. Kunnavakkam, F.M. Houlihan, M. Schlax, J.A. Liddle, P. Kolodner, O. Nalamasu, J.A. Rogers, Low-cost, low-loss microlens arrays fabricated by soft-lithography replication process, Applied Physics Letters 82 (8) (2003) $1152-1154$.

[14] C.D. Jones, M.J. Serpe, Microlens formation in microgel/gold colloid composite materials via photothermal patterning, Journal of the American Chemica Society 125 (18) (2003) 5292-5293.

[15] M. Feldmann, S. Bütefish, S. Büttgenbach, A novel electrically controlled flexible liquid microlens, in: DTIP of MEMS \& MOEMS, IEEE, Cannes-Mandelieu, 2003.
[16] N. Chronis, G.L. Liu, K.H. Jeong, L.P. Lee, Tunable liquid-filled microlens array integrated with microfluidic network, Optics Express 11 (19) (2003) 2370-2378.

[17] K.H. Jeong, G.L. Liu, N. Chronis, L.P. Lee, Tunable microdoublet lens array, Optics Express 12 (11) (2004).

[18] A.N. Simonov, O. Akhzar-Mehr, G. Vdovin, Light scanner based on a viscoelastic stretchable grating, Optics Letters 30 (9) (2005) 949-951.

[19] A. Llobera, V.J. Cadarso, K. Zinoviev, C. Domínguez, S. Büttgenbach, J. Vila, J.A. Plaza, Poly(Dimethylsiloxane) waveguide cantilevers for optomechanical sensing, IEEE Photonics Technology Letters 21 (2) (2009) 79-81.

[20] F. Carrillo, S. Gupta, M. Balooch, S.J. Marshall, G.W. Marshall, L. Pruitt C.M. Puttlitz, Nanoindentation of polydimethylsiloxane elastomers: effect of crosslinking, work of adhesion, and fluid environment on elastic modulus, Journal of Materials Research 21 (2006) 535-537.

[21] F. Schneider, J. Draheim, R. Kamberger, U. Wallrabe, Process and material properties of Polydimethyl-siloxane (PDMS) for Optical MEMS, Sensors Actuators A 151 (2009) 95-99.

[22] M. Katz, Introduction to Geometrical Optics, World Scientific, New York, USA 1994.

[23] K. Hosokawa, T. Fujii, I. Endo, Handling of picoliter liquid samples in a poly(dimethylsiloxane)-based microfluidic device, Analytical Chemistry 71 (20) (1999) 4781-4785.

[24] K. Hosokawa, K. Hanada, R. Maeda, A polydimethylsiloxane (PDMS) deformable diffraction grating for monitoring of local pressure in microfluidic devices, Journal of Micromechanics and Microengineering 12 (1) (2002) 1-6.

[25] A. Llobera, S. Demming, R. Wilke, S. Büttgenbach, Multiple internal reflection poly(dimethylsiloxane) systems for optical sensing, Lab on a Chip 7 (2007) 1560-1566.

[26] Y.N. Xia, G.M. Whitesides, Soft lithography, Annual Review of Materials Science 28 (1998) 153-184.

\section{Biographies}

Dr. Víctor J. Cadarso graduated in physics in 2003 from the Universitat Autònoma de Barcelona. In 2004 he joined the CNM-IMB (Barcelona) to perform his doctora studies in the field of integrated optics and optical MEMS in silicon and polymeric technologies. After finishing his Thesis (2009) he joined Prof. Brugger's group at EPFL where he is working in new technologies for pattern polymers focused in optical applications.

Dr. Andreu Llobera. IMB-CNM. PhD in physics from the Universitat Autònoma de Barcelona in 2002. Since 2009 he holds a permanent position as researcher at the IMB-CNM-CSIC. He has participated in several research projects financed by the Spanish Government and by the European Community, has co-authored 55 published articles, with a total of 311 citations and an h factor of eleven, he has also collaborated in the writing of three chapters of books. He holds two patents and he has contributed with 67 works (of which eight were invited) to international conferences, as well as 23 additional works to national conferences. He has been invited to give 4 seminars in foreign universities. His research activities include integrated optics devices, micro-opto-electro-mechanical systems (MOEMS) and lab-on-a-chip platforms, either using silicon and polymer technology. Finally, Dr. Llobera was awarded with a Starting Grant of the European Research Council in 2008

L.G. Villanueva graduated in physics in 2002 from the University of Zaragoza. He realized his doctoral studies in the CNM-IMB (Barcelona) within the field of NEMS/MEMS for biodetection and Scanning Probes. After finishing his Thesis (2006) he joined Prof. Brugger's group at EPFL where he was working in emerging nanofabrication techniques and mechanical sensors until 2009. He was awarded with an IOF Marie Curie Fellowship, position that is currently holding at the California Institute of Technology (Caltech). His main interests are focused on coupled and synchronization phenomena in NEMS.

J.A. Plaza (Ph.D.) was born in Cerdanyola del Vallés (Barcelona), Spain in 1968. He received his physicist degree and his $\mathrm{Ph}$. D. degree in electronics engineering from the Universidad Autónoma de Barcelona $(1992,1997)$. Since 1995, he has the degree of Specialist in simulation by the Finite Element Method from the Universidad Nacional de Educación a Distancia . Currently he is working at the Micro and Nanosystems Department at the National Institute of Microelectronics IMB-CNM (CSIC) in Cerdanyola (Barcelona), Spain. He has been focused on technology development, design, simulation and characterization of Microsystems and Nanosystems. Now, his research is focused on MEMS and NEMS for life science applications.

Juergen Brugger (Dipl. Electronique-Physique and Dr.sc. from the University of Neuchatel) joined the Ecole Polytechnique Federale de Lausanne (EPFL) in 2002 as tenure-track assistant professor within the Faculty of Engineering Science and Technology (STI). In 2008 he was appointed associated professor and vice-director of the Institute of Microtechnology (IMT). Before joining EPFL, he was at the MESA+ Research Institute of Nanotechnology, University of Twente, The Netherlands, at IBM Zurich Research Laboratory, and at the Hitachi Central Research Laboratory, Tokyo. Since 1995, he works in the field of interdisciplinary and experimental micro and nanotechnologies towards integrated micro/nanosystems with components at mesoscopic scales. In his research, he combines methods of clean-room technologies with emerging micro and nanopatterning methods, such as scanning probes, stencil 
lithography and inkjet printing, to be applied to the field of information technologies and life-sciences. Dr. Brugger has published over 90 peer-reviewed scientific publications. He is editorial board member of the journal "Nanotechnology". He served as program committee member of IEEE-IEDM, program chair of IEEE-NEMS, technical program committee of IEEE-MEMS, Hongkong 2010 and General Chair for the Eurosensors XXIII, Lausanne, 2009. His own laboratory presently consists of 4 postdocs and $6 \mathrm{PhD}$ students. $\mathrm{He}$ is co-inventor of about 10 patents and received two IBM research awards.
Carlos Domínguez received the B.S., M.S., and Ph.D. degrees in chemistry from the Universidad Complutense of Madrid, Spain, in 1980, and 1985, respectively He became a member of the scientific staff at the Instituto de Microelectrónica de Barcelona (IMB-CNM, CSIC) in 1986. Since 1991 he has been a Senior Scientific Researcher. He is involved in materials and process development for new transducers and sensors. Currently he is working on the development of an integrated optical technology based on silicon for (bio)-chemical sensors and broad-band telecommunications applications. 